\title{
Chemotherapy
}

\section{Do You Know Pixantrone?}

\author{
Giorgio Minotti Pierantonio Menna Emanuela Salvatorelli \\ Department of Medicine and Center for Drug Sciences, University Campus Bio-Medico, \\ Rome, Italy
}

Pixantrone is a novel anthracenedione, and as such it is popularly referred to as an "anthracycline-like" drug. Pixantrone has been obtained by extensive chemical modifications of the prototypic anthracenedione, mitoxantrone, and it has been approved by the European Medicines Agency (EMA), but not by the US Food and Drug Administration (FDA), for the treatment of refractory or relapsed non-Hodgkin lymphoma ( $\mathrm{r} / \mathrm{r} \mathrm{NHL})$.

For patients with newly diagnosed NHL, frontline therapy rests with an immuno-chemotherapy regimen that is called R-CHOP, where $\mathrm{R}$ reads rituximab (an anti-CD20 antibody) and CHOP reads a cocktail of cyclophosphamide, doxorubicin, vincristine, and prednisone. The reasons pixantrone was rejected by the FDA were quite multifaceted. The phase 3 study of pixantrone versus investigator-choice chemotherapeutics for the treatment of patients with $\mathrm{r} / \mathrm{r}$ NHL recruited much fewer than planned patients, and in addition, only half of the patients were recruited in countries where rituximab was available for combination with $\mathrm{CHOP}$ at the time when NHL was diagnosed and CHOP was commenced. Thus, the FDA felt that the registration trial was both underpowered and structurally inadequate to provide a real representation of patients with $\mathrm{r} / \mathrm{r}$ NHL. Further concerns were due to the incidence of grade 3-4 cardiotoxicity, which seemed to be higher in the pixantrone arm compared to investigator-choice drugs, as if pixantrone retained an anthracycline-like cardiotoxicity and precipitated subclinical cardiac damage induced by the doxorubicin-containing CHOP [1].

\section{KARGER}

(C) 2017 S. Karger AG, Basel

E-Mail karger@karger.com

www.karger.com/che
Following approval by the EMA, which maintained a role for pixantrone in a difficult-to-treat condition like $r / r$ NHL, the drug became available in many countries, but its popularity with hematologists remained disappointing to say the least. It goes without too many words that the FDA decision counts, particularly in days when new drugs are running in the pipeline and hold promise for important twists in the field. Many doctors actually do not know pixantrone, and if they do, they believe it is one more anthracycline. In this issue of the journal, Appio et al. [2] report on a clinical case that clearly shows the might of pixantrone. They describe the case of a 56-year-old NHL patient who relapsed after R-CHOP, underwent second-line salvage therapy with rituximab, dexamethasone, high-dose cytarabine, and cisplatin (or oxaliplatin), but progressed before autologous stem cell transplantation could be performed. Third-line therapy with pixantrone and a single dose of rituximab offered a complete response and allowed the doctors to submit the patient to successful highdose chemotherapy, followed by autologous stem cell transplant and involved-field consolidation radiotherapy as appropriate. The patient enjoys a remission longer than a year, and his cardiac function is fully preserved. Thus, pixantrone was an excellent tool to bridge the patient to transplant - not bad for a little-known drug.

This is a case report and to be honest with our readers, we must recognize that one swallow does not make a summer. This having been acknowledged, the report by Appio et al. [2] raises a number of positive issues. The

Prof. Giorgio Minotti

Department of Medicine and Center for Drug Sciences, University Campus Bio-Medico Via Alvaro del Portillo 21

IT-00128 Rome (Italy)

E-Mail g.minotti@unicampus.it 
doctors were brave enough to use a drug that has not yet obtained the reimbursement waiver by the Italian regulatory authority, they were rigorous enough to use pixantrone in full compliance with its approval status, and they were diligent enough to check the patient's cardiac function before and after pixantrone therapy. The doctors also knew that pixantrone is active in $\mathrm{r} / \mathrm{r}$ NHL due to mechanisms of action that are different from those of anthracyclines (less evident inhibition of topoisomerase II a versus more vigorous effects on alkylating DNA and perturbing mitotic fidelity in cancer cells) [3]. What the doctors omitted to emphasize is that the apparent cardiac safety of pixantrone, as documented for their high-risk patient, can be traced also in the underrated phase 3 study. In that study the higher incidence of grade 3-4 cardiotoxicity was largely caused by an unbalanced randomization process, such that more patients with pre-existing and ongo- ing cardiac disease were randomized to pixantrone [1]. Were these patients eliminated from trial analysis, pixantrone would show superior to investigator-choice drugs without introducing an excess risk of cardiotoxicity. In a limited phase $2 \mathrm{~B}$ study that probed $\mathrm{R}-\mathrm{CHOP}$ versus an $\mathrm{R}-\mathrm{CPOP}$ regimen in which pixantrone was substituted for doxorubicin, pixantrone was active and caused less cardiac toxicity than doxorubicin [4]. Thus, pixantrone is intrinsically less cardiotoxic than doxorubicin and can safely be administered to patients with a history of prior anthracycline. Pharmacologic determinants of the cardiac safety of pixantrone have been described [5] and can be summarized as follows: pixantrone looks like an anthracycline but it is not $[3,6]$.

The work by Appio et al. [2] may help to improve the awareness of hematologists of the therapeutic opportunities pixantrone may serve in the settings of $r / r$ NHL.

\section{References}

1 Pettengell R, Coiffier B, Narayanan G, et al: Pixantrone dimaleate versus other chemotherapeutic agents as a single-agent salvage treatment in patients with relapsed or refractory aggressive non-Hodgkin lymphoma: a phase 3 , multicentre, open-label, randomized trial. Lancet 2012;13:696-706.

2 Appio L, Landoni C, La Targia M, Bertolli V, Chiarucci M, Crovetti G, Vassenna E, Serio G, Bregni M: Single-agent pixantrone as a bridge to autologous stem cell transplantation in a patient with refractory diffuse large B-cell lymphoma. Chemotherapy 2017;62:187-191.
3 Menna P, Salvatorelli E, Minotti G: Rethinking drugs from chemistry to therapeutic opportunities: pixantrone beyond anthracyclines. Chem Res Toxicol 2016;29:1270-1278.

4 Herbrecht R, Cernohous P, Engert A, et al: Comparison of pixantrone-based regimen (CPOP-R) with doxorubicin-based therapy (CHOP-R) for treatment of diffuse large Bcell lymphoma. Ann Oncol 2013;24:26182623.
5 Salvatorelli E, Menna P, Paz OG, et al: The novel anthracenedione, pixantrone, lacks redox activity and inhibits doxorubicinol formation in human myocardium: insight to explain the cardiac safety of pixantrone in doxorubicin-treated patients. J Pharmacol Exp Ther 2013;344:467-478.

6 Salvatorelli E, Menna P, Cantalupo E, et al: The concomitant management of cancer therapy and cardiac therapy. Biochim Biophys Acta 2015;1848:2727-2737. 